Ann. Zootech., 1975, 24 (2), 299-310.

\title{
EFFETS DE DIFFÉRENTS NIVEAUX DE RESTRICTION HYDRIQUE SUR L'INGESTION D'ALIMENTS SOLIDES PAR LE LAPIN
}

\author{
M. PRUD'HON, Michaëlle CHÉRUBIN, Y. CARLES \\ et J. GOUSSOPOULOS \\ Station de Physiologie animale, I. N.R. A., \\ E. N.S. A., 9, Place Viala, \\ 34060 Montpellier Cedex
}

\section{RÉSUMMÉ}

Nous avons cherché à mesurer l'influence d'une réduction de la période d'abreuvement sur les caractéristiques de la consommation d'aliments solides de lapins mâles Néo-Zélandais d'âges différents élevés en cage individuelle à $20 \pm \mathrm{I}^{\circ} \mathrm{C}$.

Dans un premier essai, 3 lapins âgés de 23 semaines, dont le comportement alimentaire était connu de façon détaillée, ont reçu de l'aliment solide à volonté et de l'eau pendant une période limitée à ro minutes par jour puis Io minutes tous les deux jours. Un enregistrement graphique continu des consommations a été pratiqué pendant une période de 45 jours et l'on a étudié l'évolution de la fréquence des repas et des quantités consommées à la suite des deux types de restriction pratiqués.

Dans un deuxième essai, mené simultanément, nous avons étudié les répercussions de ces mêmes restrictions sur la vitesse de croissance et la consommation d'aliments de I 2 jeunes lapins en croissance ( 6 à I 2 semaines) et I 2 futurs reproducteurs (I I à I 7 semaines).

La réduction de la durée d'abreuvement pratiquée entraîne une chute importante des consommations au cours des premiers jours du traitement suivie d'une adaptation progressive lorsque l'allocation d'eau est quotidienne. Les repas sont fréquents et les quantités d'eau consommées élevées au cours des 2 à 3 premières heures suivant la distribution d'eau; en dehors de cette période l'aspect préférentiellement nocturne du comportement alimentaire du Lapin n'est pas aboli.

Le niveau d'ingestion d'aliments, après adaptation, est inférieur à la normale de 14 à 24 p. IoO avec une distribution d'eau quotidienne; de 30 à 46 p. Ioo avec une distribution d'eau un jour sur deux. Ces réductions de consommation sont significatives; elles sont plus marquées chez les sujets les plus âgés.

La. vitesse de croissance n'est pas significativement réduite avec une distribution d'eau quotidienne par contre une restriction plus sévère entraîne un ralentissement de croissance chez les sujets les plus jeunes, un arrêt de croissance ou une chute de poids chez les lapins les plus âgés.

Les possibilités d'utilisation pratique d'une restriction hydrique pour abaisser le niveau de consommation du Lapin sont évoquées. 


\section{INTRODUC'TION}

Chez le Lapin domestique comme dans de nombreuses autres espèces il existe une relation étroite entre les niveaux d'ingestion d'aliments solide et d'eau de boisson malgré l'existence de différences entre individus assez sensibles.

Selon les travaux de Cizek (I96r), Prud'hon (ig67), Prud'hon to al. (r974) la quantité d'eau ingérée est environ deux fois supérieure à la matière sèche consommée mais peut subir des variations avec l'âge de l'animal, son état physiologique ou les conditions climatiques. De nombreuses observations réalisées dans les élevages lorsque les dispositifs d'abreuvement sont insuffisants ou déficients permettent de conclure que le Lapin subissant une restriction hydrique mange peu; il a une croissance perturbée. Cependant ces observations sont rarement chiffrées.

Dans le cadre d'une étude plus générale sur le comportement alimentaire du Lapin domestique (Prud'hon et al., I972 ; Prud'hon, Carles et Goussopoulos, I973; ChERUBIN, I974) nous nous sommes proposé d'étudier l'influence d'une restriction hydrique plus ou moins sévère sur les différents paramètres de la prise d'aliments solides du Lapin; nous nous sommes demandé également s'il était possible, par le biais d'une restriction hydrique bien mesurée, de limiter le niveau de consommation et d'éviter le gaspillage d'aliments des lapins à l'engraissement et des futurs reproducteurs alimentés ad libitum. Nous rapportons ici les premiers résultats obtenus.

\section{MATÉRIFL ET MÉTHODES}

\section{Essai 1}

Trois lapins mâles Néo-Zélandais, âgés de $\mathrm{I} 62$ jours, utilisés précédemment pour l'étude du comportement alimentaire du lapin en croissance, ont été maintenus pendant 45 jours dans des cages munies du dispositif d'enregistrement graphique des consommations décrit précédemment (Prun'hon, Carles et Goussopoulos, 1973).

Ils recevaient à volonté un aliment granulé commercial dosant i 5 p. Ioo de matières azotées totales et I4 p. Ioo de cellulose. La distribution d'eau était assurée par un abreuvoir à clapet, alimenté en permanence pendant la période pré-expérimentale (de $J_{0}$ à $J_{14}$. De $J_{14}$ à $J_{30}$ l'eau était distribuée pendant io ninutes par jour, de $2 \mathrm{I}$ heures à $2 \mathrm{I} \mathrm{h} \mathrm{I} O$, une heure après l'allumage du bâtiment; de $\mathrm{J}_{30}$ à $\mathrm{J}_{45}$ la distribution d'eau n'était plus assurée qu'un jour sur deux pendant Io minutes.

La température était constante : $20 \pm \mathrm{I}^{\circ} \mathrm{C}$ et la durée d'éclairement de $\mathrm{I} 4$ heures par jour réparties de 20 heures à ro heures.

Les consommations d'aliments solides (poids des prises et répartition dans le temps) ont été enregistrées pendant toute la durée de l'essai ; la consommation d'eau n'a pu être mesurée de façon précise sur les sujets expérimentaux compte tenu du gaspillage survenant chez les lapins soumis à restriction pendant la période de disponibilité de la boisson; une évaluation des quantités bues, par pesée avant et après absorption d'eau, a été réalisée chez des sujets de même âge placés dans les mêmes conditions expérimentales mais non soumis à enregistrement.

Les lapins ont été pesés au début et à la fin de chaque période expérimentale ainsi qu'une fois par semaine, le lundi entre 9 heures et ro heures.

\section{Essai 2}

Vingt-quatre lapins mâles Néo-Zélandais blancs âgés de 6 semaines (1 2 lapins) et I I semaines ( 2 lapins) ont été répartis en quatre lots équilibrés, deux lots témoins ct cleux lots expérimentaux, 
placés en cages individuelles dans une pièce maintenuc à température constante $\left(20 \pm \mathrm{I}^{\circ} \mathrm{C}\right)$. $\mathrm{La}$ durée d'éclairement était de $\mathbf{4}$ heures par jour et l'intensité lumineuse d'environ Io lux au niveau des cages

Le lot témoin a reçu le même aliment granulé du commerce que dans l'essai I et de l'eau de boisson à volonté pendant 50 jours.

Les lapins des lots expérimentaux ont, après une semaine d'acclimatation, été restreints dans leur consommation d'eau. Pendant les trois premières semaines ils ont́ disposé d'eau de boisson Io minutes par jour (de ro heures à ro h ro) au cours des trois semaines suivantes la distribution d'eau était réaliséc un jour sur deux. L'aliment granulé était distribué à volonté pendant toute la période expérimentale.

Les Iapins et les aliments étaient pesés deux fois par semaine après la période d'abreuvement.

\section{RÉSULTATS}

\section{I. - Restriction hydrique des lapins en fin de croissance}

a) Comportement des lapins.

Les lapins privés de boisson pendant 24 heures ou 48 heures boivent sans discontinuer pendant les 5 ou 6 premières minutes de distribution d'eau puis se mettent à manger. Ia quantité d'eau consommée d'affilée est, pour les sujets que nous avons étudiés, de I62,8 $\pm 27 \mathrm{ml}$ pour le lot recevant de l'eau chaque jour et $15 \mathrm{I} \pm 30 \mathrm{ml}$ pour le lot abreuvé tous les deux jours cette différence n'étant pas significative.

TABIEAU I

Consommation journalière d'aliment de lapins disposant d'eau ad libitum puis rationnés ( ${ }^{1}$ )

(Essai I)

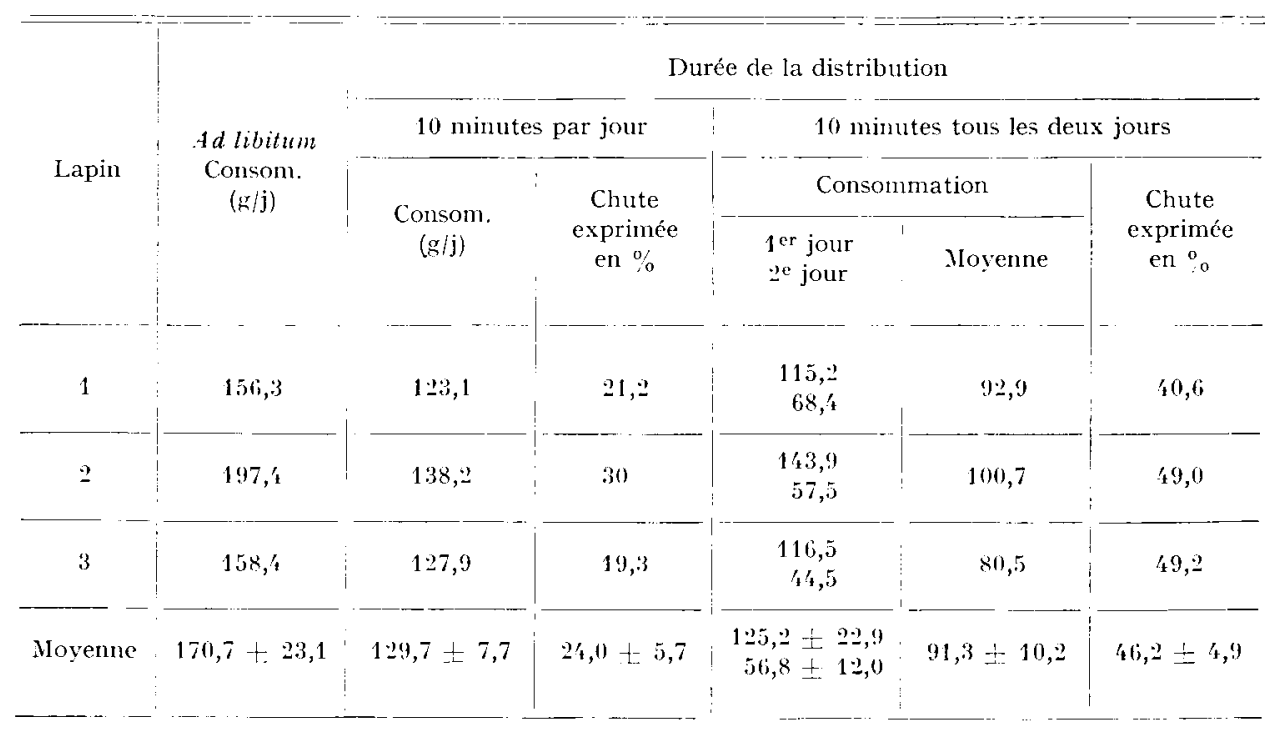

(1) Ces moyennes ont été établies sans tenir compte des résultats des huit premiers jours au cours desquels se produit l'adaptation. 

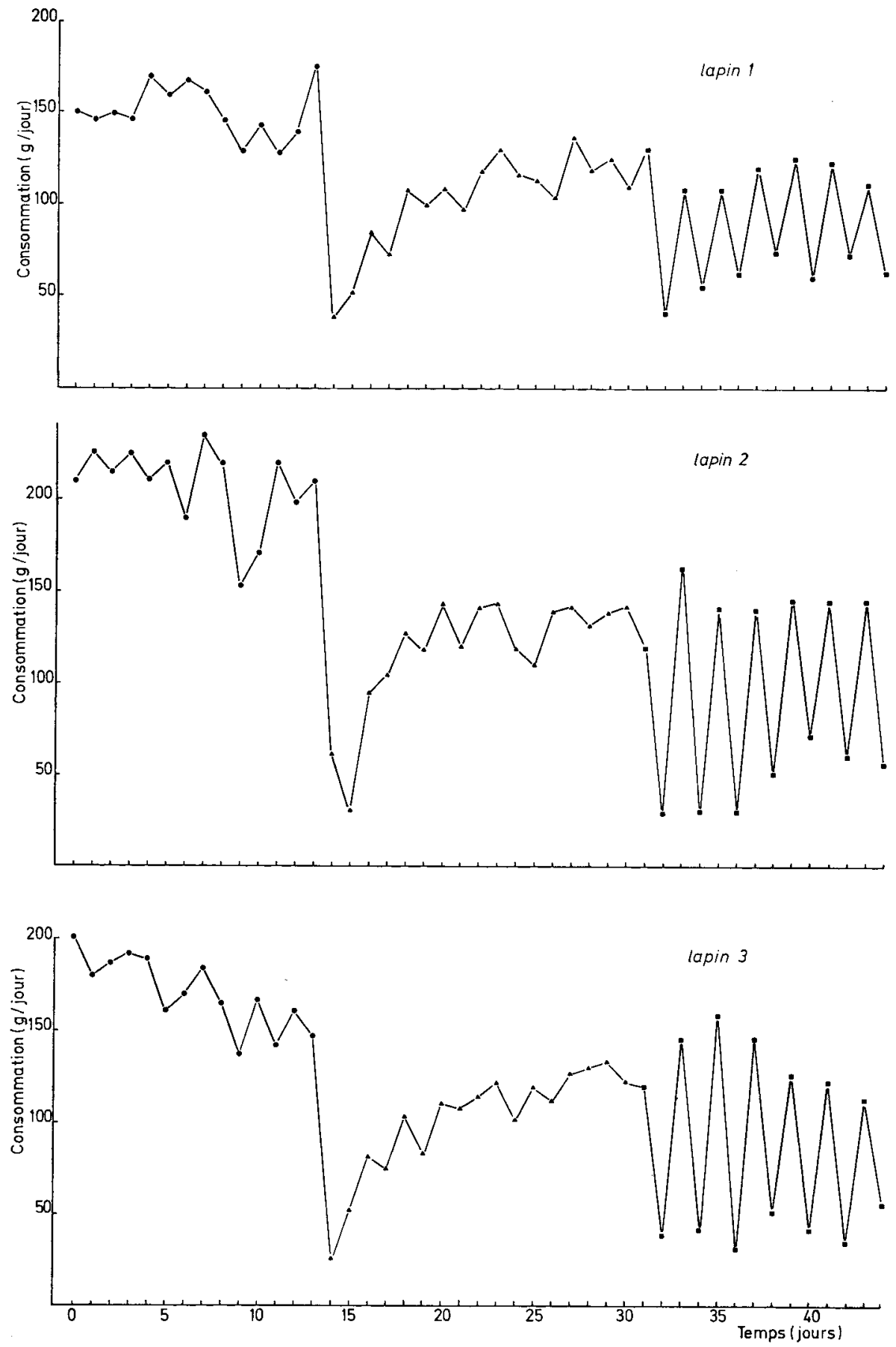

FIG. I. - Evolution de la consommation quotidienne d'aliments solides de trois lapins abreuvés ad libitum puis soumis à deux niveaux de restriction hydrique - - eau ad libitum

- eau ro minutes tous les deux jours 


\section{b) Niveau de consommation.}

Sur la figure I sont représentées, pour les trois lapins enregistrés, les courbes de consommation journalière d'aliment au cours de la période expérimentale. La réduction de disponibilité de l'eau entraîne une chute brutale de consommation d'aliment qui s'atténue peu à peu, le Lapin s'adaptant à ses nouvelles conditions d'alimentation en une dizaine de jours. Toutefois (tabl. I) le niveau de consommation ne redevient pas normal ; la baisse de consommation est de 1'ordre de $25 \mathrm{p}$. Ioo. La distribution d'eatı ro minutes toutes les $4^{8}$ heures entraîne une consommation en dents de scie, le Lapin mangeant peu le jour de jeûne hydrique et ne compensant pas cette restriction par une surconsommation le jour suivant (tabl. I). En moyenne la baisse de consommation est de l'ordre de $46 \mathrm{p}$. Ioo par rapport à la consommation normale.

Le passage d'un régime d'abreuvement ad libitum à un abreuvement ro minutes par jour entraîne un ralentissement ou un arrêt de croissance (tabl. 2), un abreuvement tous les deux jours entraîne une perte de poids.

\section{TABLEAU 2}

Fuolution pondérale des lapins soumis aux essais de rationnement hydrique

(lissai I)

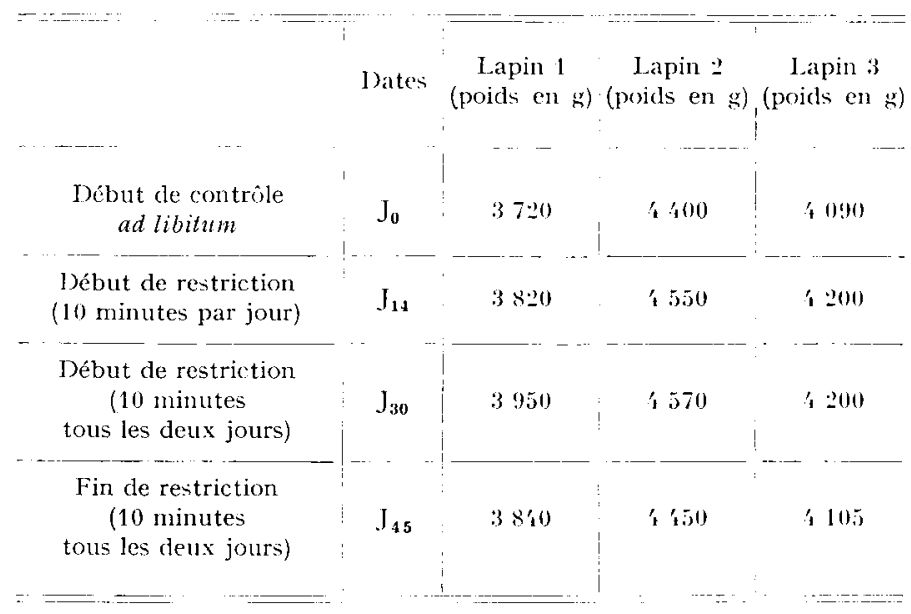

\section{c) Fréquence des repas et nivean de consommation.}

Sur les figures $2 a$ et $2 b$ nous avons représenté les polygones de distribution des fréquences et des consommations moyennes horaires au cours d'une période de 48 heures. Chez les lapins recevant de l'eau ad libitum. Le nombre de prises alimentaires et la quantité d'aliment consommée s'abaissent au cours des prenières heures d'éclairement puis s'élèvent au cours des heures précédant l'extinction des feux et restent à un niveau élevé pendant les heures d'obscurité.

Chez les lapins soumis à une restriction hydrique la distribution d'eau est suivie d'une période de 2 à 3 heures d'intense consommation d'aliments ; en dehors de cette période, l'activité alimentaire est liée au rythme d'éclairement du local d'une façon tout à fait comparable aux lapins témoins. 
Il semble que la fréquence des prises soit sensiblement moins modifiée par la durée de la diète hydrique que ne l'est le niveau de consommation : au bout de 36 heures les prises d'aliment restent fréquentes mais de faible importance. Notons encore que les lapins habitués à recevoir de l'eau tous les jours et à consommer immédiatement après continuent à effectuer des repas supplémentaires à la même heure lorsqu'on supprime la boisson un jour sur deux.
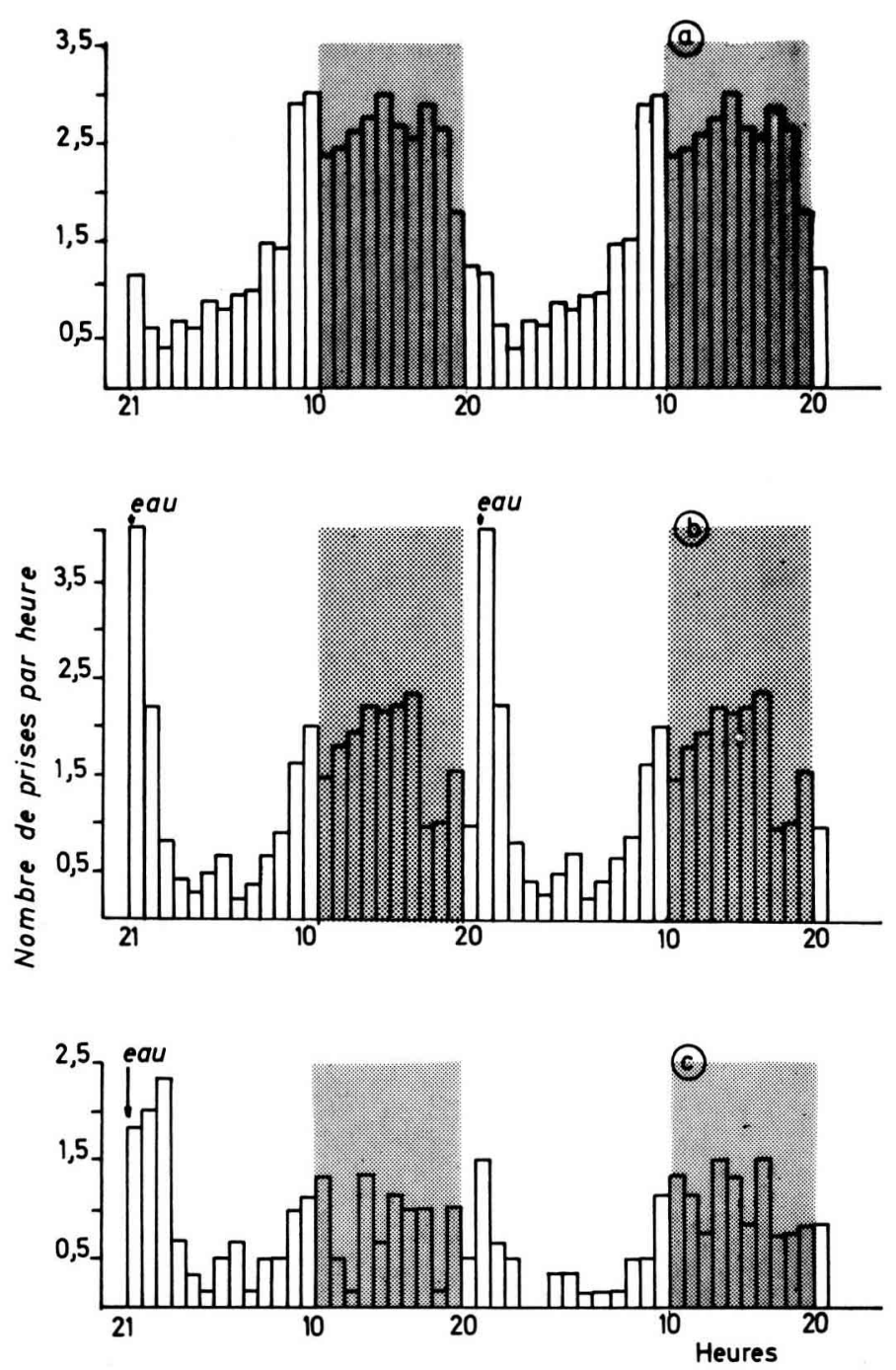

Fici. $2 a$. - Evolution des fréquences moyennes horaires des repas an cours d'une période de 48 heures cher les lapins abrewves ad libitum puis soumis à deux niveaux de réduction de la duréc d'abreutement

$$
\begin{aligned}
& a: \text { Eau ad libitum } \\
& b \text { : Eau ro minutes par jour } \\
& c \text { : Eau ro minutes tous le's deux jours }
\end{aligned}
$$


(a)

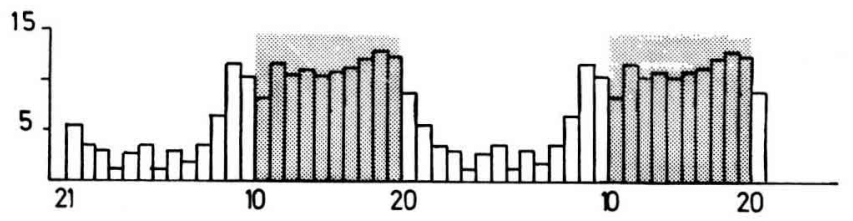

(b)

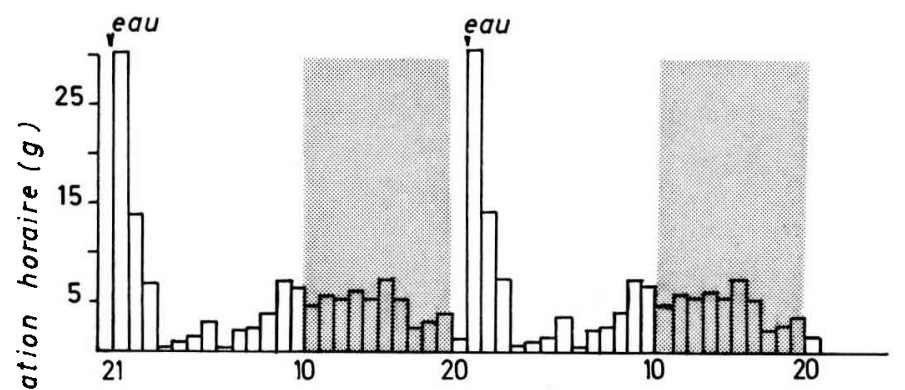

(c)

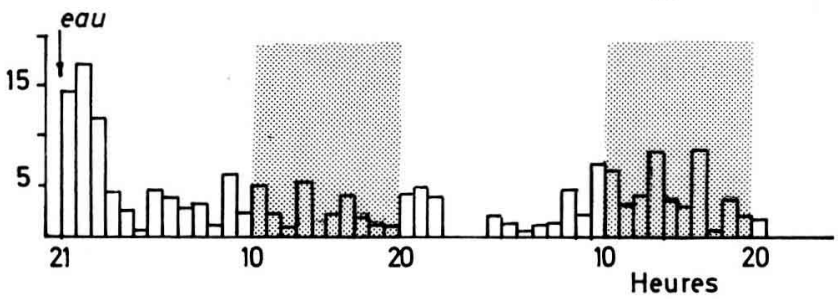

Fig. 2 b. - Evolution des consommations moyennes horaires an cours d'une période de 48 heures chez les lapins abreuvés ad libitum puis soumis à deux niveaux de réduction de la durée d'abreuvenent

$a$ : liau ad libitum

$b$ : Jau ro minutes par jour

$c$ : Eau ro minutes tous les cleux jours

2. - Restriction hydrique des lapins en croissance

L'effet d'une restriction hydrique d'abord modérée (Io minutes d'eau par jour) puis sévère (ro minutes d'eau tous les deux jours) n'a pas été identique selon qu'elle était appliquée à des lapins de boucherie en pleine croissance (de 6 à I2 semaines) ou à de futurs reproducteurs plus âgés (de II à I7 semaines).

Sur la figure 3 sont représentées les courbes de croissance moyenne des différents lots expérimentaux : 

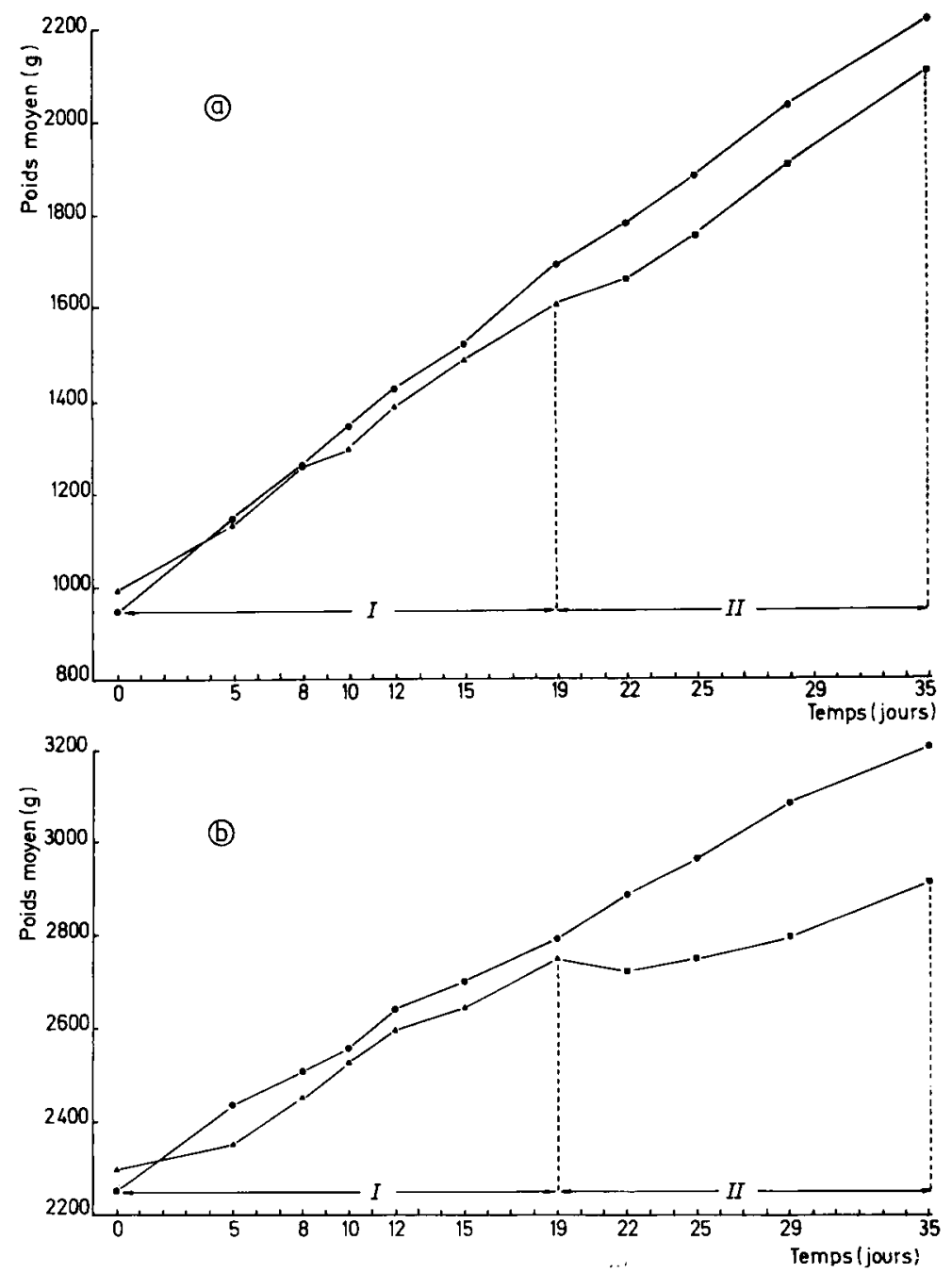

líg. 3. - Evolution ponterale de jounes lapins cn croissance (a) et de futurs reproductcurs (b) soun is ou non à differcnts niveaut successifs de vestriction hydrique

- - eau à volonté

- A call ro minutes par jour

- - caulo minutes tous les deux jours

La croissance de; sujets les plus jeunes n'est pas affectée par une distribution d'eau quotidienne ; elle est ralentie d'une façon significative (tabl. 3) par une distribution tous les deux jours.

Les sujets plus âgés voient leur croissance réduite de façon non significative par une restriction modérée et de façon hautement significative par une restriction sévère.

La consommation d'aliments est dans tous les cas significativement plus faible chez les sujets restreints, l'abaissement du niveau d'ingestion étant respectivement 


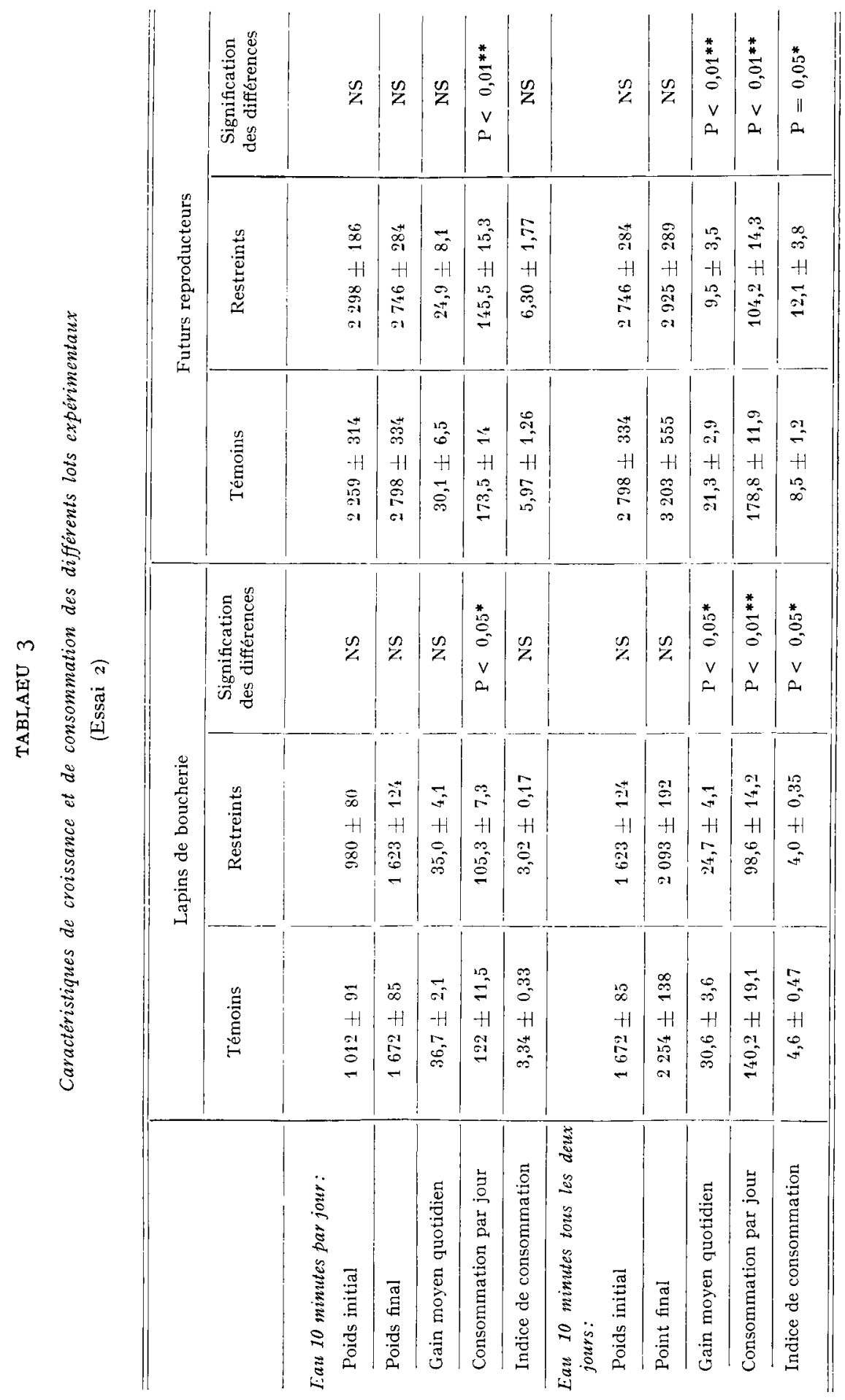


de I4 p. Ioo (jeunes, Io mm/24 h), 30 p. Ioo (jeunes, Io mn/48 h), I6 p. Ioo (âgés, Io $\mathrm{mn} / 24 \mathrm{~h}$ ), $42 \mathrm{p}$. Ioo (âgés Io $\mathrm{mn} / 48 \mathrm{~h}$ ). Ces valeurs sont plus faibles que dans l'essai I ot nous utilisons des lapins proches de leur poids adulte. L'indice de consommation a tendance à être inférieur chez les jeunes restreints et supérieur chez les futurs reproducteurs restreints ; la différence est significative lors d'une restriction d'abreuvement sévère.

\section{DISCUSSION}

La réduction de la période de disponibilité de l'eau entraîne, à très court terme, une perturbation sensible de la consommation d'aliments solides suivie d'une certaine adaptation et accompagnée d'une modification durable du comportement alimentaire et du niveau de consommation.

La période de distribution de l'eau avait été située au cours de la deuxième heure d'éclairement à une époque où normalement la consommation devient très faible (Prud'hon, Carles, Goussopoulos, i973 ; Prud'hon et al., I974). Malgré cela le jeûne hydrique a induit une forte activité alimentaire pendant les heures suivant la distribution d'eau ; toutefois l'activité alimentaire nocturne est loin d'avoir été abolie: chaque nuit la fréquence des prises d'aliments s'élève même lorsque la distribution d'eau n'a lieu que tous les deux jours.

La restriction du temps d'accès à l'eau entraîne une restriction de consommation d'aliment ; cet effet est d'autant plus marqué que la durée du jeûne est plus élevée ce qui n'est pas étonnant dans la mesure où après un jê̂ne hydrique de 48 heures le Lapin ne boit pas plus en Io minutes qu'après un jêtne de 24 heures. Cizer (I96I) a montré que après trois jours de jeûne hydrique le Lapin ne consommait plus que 2 p. Ioo de sa ration ordinaire puis il s'arrête de manger. Toutefois, selon Hayward (I96I), il possède une grande faculté de résistance à la déshydratation et peut survivre jusqu'à avoir réduit son poids de moitié.

L'effet de la restriction hydrique est d'autant plus marqué que les sujets restreints sont plus âgés ; les baisses de consommation sont respectivement de 14 , I 6 et 24 p. Ioo chez les lapins de 8 , I 2 et 26 semaines auxquels on alloue Io minutes d'abreuvement par jour - les baisses correspondantes étant de 30,42 et $46 \mathrm{p}$. Ioo avec une allocation tous les deux jours. Les résultats demeurent à confirmer compte tenu du faible effectif étudié et nous n'en avons pas analysé 1'origine. Notons simplement que le développement de l'estomac, dont pourrait dépendre la quantité d'eau consommée au cours des ro minutes d'abreuvement, est en valeur relative, plus élevée chez les sujets de 8 semaines que chez les sujets plus âgés (CANTIER et al., I969; LEBAs et LAPLACE, I972). En outre nous avons montré (PRUD'HON et al., I974) chez des lapins alimentés ad libitum que le rapport eau de boisson/matière sèche ingérée augmentait avec l'âge passant de $I, 9$ à 6 semaines à 2,2 à $I 8$ semaines. On peut donc supposer qu'à consommation d'eau équivalente les lapins jeunes pourront ingérer davantage de matière sèche que les sujets plus âgés. 


\title{
CONCLUSION
}

Au niveau de l'évolution pondérale, une restriction hydrique d'une durée de 2.4 heures n'affecte pas la croissance des jeunes lapins de boucherie; la consommation étant abaissée il en est de même de l'indice de consommation; elle affecte peu la croissance des futurs reproducteurs une fois passée la période d'adaptation. Une restriction plus sévère affecte la croissance des deux catégories de lapins d'une façon continue, plus marquée chez les animaux les plus âgés.

La restriction de la durée d'abreuvement des lapins entraîne une modification de l'horaire des consommations ainsi qu'une restriction concommitante des quantités ingérées dont l'importance est fonction, dans un milieu maintenu à température constante, de l'intervalle entre les périodes d'abreuvement et de l'âge des animaux.

D'un point de vue pratique ces essais mettent bien en évidence l'influence néfaste d'un arrêt d'abreuvement accidentel sur la consommation d'aliments du Lapin; en sens inverse il paraît possible, par un choix judicieux de l'horaire de distribution de la boisson, d'assurer une croissance correcte aux lapereaux à l'engrais tout en restreignant le niveau de consommation des futurs reproducteurs et même des lapins en gestation puisque LEBAS (1974) a montré qu'un tel rationnement est bénéfique pour le démarrage de la lactation ultérieure.

Ceci représente une manière originale de restreindre la consonmation tout en conservant une distribution ad libitum.

Il reste :

- à vérifier la validité de cette technique lorsqu'on travaille à plus grande échelle dans des conditions de milieu moins maîtrisées, sur des sujets élevés en groupe ;

- à préciser les caractéristiques d'abreuvement les plus souhaitables pour chaque type de lapin;

- à rechercher les effets à long terme d'un tel type de traitement sur la santé et la productivité des animaux.

$$
\text { Recu pour publication en janvier } 1975 .
$$

\section{SUMMARY}

\author{
EFIECTS OF DIFFERENT WATER RESTRICTION LEVEIS \\ ON THF: FOOD INTAKE OF RAIBBITS
}

Juring the present study, an attempt was made to determine the influence of reduced drinking time on fool intake characteristics of male $N$ ew Zealand rabbits of different ages kept in individual cages at $20 \pm 1{ }^{\circ} \mathrm{C}$.

In the first trial, three 23 weeks old rabbits, whose fecding behaviour was well established, received food ad libitum and water for a linited period of 10 minutes per day, and thereafter for Io minutes every two days. A continuous graph recording of the consumptions was made for a period of 4.5 clays and the variation in the frequency of meals and in the amounts ingested when practising these two types of water restriction, was studied. 
In the second trial, performed simultaneously, the repercussions of the same restrictions on the growth rate and food intake were studied in 12 young growing rabbits (6-I 2 weeks) and in I 2 future breeding animals (I I-I 7 weeks).

Reduction of the drinking time brought about a large decrease in food intake cluring the first few days of the treatment, followed by a progressive adaptation while there was a daily allowance of water. The meals were frequent and the amounts of water ingested were high during the first 2-3 hours following the administration of the water. Except for this period, the preferentially nocturnal aspect of the rabbit's feeding behaviour was not abolished.

After the adaptation, the food intake level was $\mathrm{I}_{4}$ to $24 \mathrm{p}$. I 00 below the normal values when the water was allotted every two days. These reductions in the consumptions were significant and more marked in older animals.

The growth rate was not significantly reduced with a daily administration of water. However, a more severe restriction led to lower growth rates in the younger animals and a ccasing of growth or a weight decrease in the older rabbits.

The possibilities of practical utilization of water restriction to reduce the food intake level in rabbits, are mentionned.

\section{RÉFÉRENCES BIBLIOGRAPHIQUES}

Canter J., Vezinhet A., Rouvinz R., Dal"zier L., I969. Allométrie de croissance chez le Lapin (oryctolagus cuniculus). I. Principaux organes et tissus, Ann. Biol. anim. Bioch. Biophys., 9, 5-39.

Chertin M., 1974. Etude du comportement alimentaire du Lapin domestique du sevrage à la dix-huitieme scmaine. Infuence de quelques facteurs extrinsiques sur les paramitres de l'ingestion alimentairc. Thèse I)r. Spécialité. Montpellier.

Cizer L. J., I96r. Relationship between food and water ingestion in the rabbit. Am. J. Physiol., 201, $557-566$.

HAYWARD J. S., rg6r. The ability of the wild rabbit to survive conditions of water restriction. C. S. I. R. O. Wildl. Res., 6, 160-175.

Lebas F., r975. Étude chez la Lapine de l'influence du niveau d'alimentation durant la gestation. I. Sur les performances de reproduction. Ann. Zootcch., 24, 267-279.

Lebas F., Laplace J. P., 1972. Mensurations viscérales chez le Iapin, I. Croissance du foie, des reins et des divers segments intestinaux entre 3 et I semaines d'âge. Ann. Zootech., 21, 37-47.

Prud'hon M., I967. L'appétit du Lapin alimenté à sec. Bull. tech. inf. ingrs. servs. agric., 29 , I-I6.

Prudiox M., Carles Y., Govssopollos J., Tiozhl P. F., 1972. Innregistrement graphique des consommations d'aliments solide et liquide du Lapin donestique nourri ad libitum. Ann. Zootech., 21, 45 I-460.

Pred'hox M., Carles Y., Goussopollos J., r973. Le conportement alimentaire du Lapin en croissance. Mise au point d'une méthode d'étude et premières observations. Congr. int. de Cuniculture, Conte, sept. 1973 .

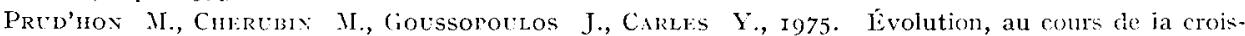
sance, des caractéristiques de la consommation d'aliments solide et liquide du Jiapin clomestique nourri ad libitum. . Inn. Zootech., 24, $289-298$. 